\title{
Viral Cre-LoxP tools aid genome engineering in mammalian cells
}

\author{
Ranjita Sengupta ${ }^{1}$, Amy Mendenhall ${ }^{1}$, Nandita Sarkar ${ }^{2}$, Chandreyee Mukherjee $^{1}$, Amirali Afshari ${ }^{1}$, \\ Joseph Huang ${ }^{1}$ and Biao Lu $^{3 *}$
}

\begin{abstract}
Background: Targeted nucleases have transformed genome editing technology, providing more efficient methods to make targeted changes in mammalian genome. In parallel, there is an increasing demand of Cre-LoxP technology for complex genome manipulation such as large deletion, addition, gene fusion and conditional removal of gene sequences at the target site. However, an efficient and easy-to-use Cre-recombinase delivery system remains lacking.

Results: We designed and constructed two sets of expression vectors for Cre-recombinase using two highly efficient viral systems, the integrative lentivirus and non-integrative adeno associated virus. We demonstrate the effectiveness of those methods in Cre-delivery into stably-engineered HEK293 cells harboring LoxP-floxed red fluorescent protein (RFP) and puromycin (Puro) resistant reporters. The delivered Cre recombinase effectively excised the floxed RFP-Puro either directly or conditionally, therefore validating the function of these molecular tools. Given the convenient options of two selections markers, these viral-based systems offer a robust and easy-to-use tool for advanced genome editing, expanding complicated genome engineering to a variety of cell types and conditions.

Conclusions: We have developed and functionally validated two viral-based Cre-recombinase delivery systems for efficient genome manipulation in various mammalian cells. The ease of gene delivery with the built-in reporters and inducible element enables live cell monitoring, drug selection and temporal knockout, broadening applications of genome editing.
\end{abstract}

Keywords: Cre-LoxP, Cre recombinase, Lentiviral vector, AAV vector, Genome editing, Talen

\section{Background}

Robust targeted nucleases, including meganuclease, zinc finger nucleases (ZFN), transcription activatorlike effector nucleases (TALEN) and clustered regularly interspaced short palindromic repeat-associated nuclease Cas9 (CRISPR-Cas9), are providing modern tools for genome engineering [1-4]. Remarkably, targeted nucleases not only can be programmed to bind specific gene loci within a complex mammalian genome, but they can also generate bi-allelic changes via their nuclease activities $[5,6]$. This new capability allows efficient generation of knockout animals and works directly on a variety of species including human cells and disease cell lines [7-10]. In fact, in combination with homologous recombination, targeted

\footnotetext{
* Correspondence: blu2@scu.edu

${ }^{3}$ Department of Bioengineering, Santa Clara University, 500 El Camino Real,

Santa Clara, CA 95053, USA

Full list of author information is available at the end of the article
}

nucleases have been used for genome editing to elucidate molecular mechanisms of many biological and disease processes using cultured cell models [5, 8]. By themselves alone, however, targeted nucleases can only produce double stranded DNA breaks, which in turn stimulate error-prone non-homologous end- joining (NHEJ), resulting in small sequence changes, termed indels, near the binding sites $[11,12]$. In combination with Cre-LoxP technology, the targeted nucleases could potentially be used in advanced and complicated genome editing to generate large and precise changes such as inframe fusion or single-nucleotide corrections [10, 13-19].

Cre-LoxP is a site-specific recombinase technology, used to carry out deletions, insertions, and inversions at specific sites in transgenic mice [20-23]. Cre-LoxP consists of a nuclease, the Cre Recombinase derived from P1 bacteriophage, which recognizes and catalyzes recombination between two LoxP recognition sites [20, 24]. Each LoxP site consists of a 34 bp consensus sequence with an 8 bp 
core spacer sequence flanked on either side by a $13 \mathrm{bp}$ palindromic sequence $[25,26]$. When a DNA element is flanked by two LoxP sites, the Cre recombinase recognizes the lox P sites and cuts it. The DNA element that is present between the two sites gets removed and the flanking sites get fused together. The orientation and location of the LoxP sites determine whether Cre recombination induces a deletion, inversion, or chromosomal translocation [21,27]. These predictable changes can be utilized to generate conditional knockouts as well as fusion reporters to study gene function and regulation. Traditionally, the Cre-LoxP system is a mainstay method for generating conditional knockouts in mice [27]. Through homologous recombination, LoxP sequences are first introduced into the animal genome flanking the gene of interest. Subsequent selection and production of homozygous off-spring allow conditional knockouts via the expression of Crerecombinase. Although the Cre-LoxP system is used routinely in transgenic animals, its application in cultured mammalian cells coupled with targeted nucleases has grown rapidly in recent years $[10,17,28,29]$. This is because Cre and targeted nucleases (ZFN, TALEN, and Cas9) have different advantages and disadvantages depending on applications $[19,26]$. Cre is restricted in editing LoxP-flanked sequences, but its predictability, specificity and robustness in removing or reversing the floxed sequences could not be easily achieved by the targeted nucleases due to a lack of both specificity and precision of these targeted nucleases [3]. Therefore, adding of Cre will allow additional genome editing ability and enable complicated and advanced genome manipulation especially when floxed sequences are present [19]. However, a lack of robust and easy-to-use Cre-delivery system imposes a great hindrance to its implementations. However, a lack of robust and easy-to-use Cre-delivery system imposes a great hindrance to its implementations.

To address this issue, we combined the Cre-LoxP gene editing technology with two very powerful viral gene delivery methods, the lentiviral and adeno associated viral (AAV) technologies. Both these viral systems have been demonstrated to deliver transgenes into a vast variety of mammalian cell types with distinctive characteristics [30-32]. The integrative nature makes lentiviral delivery highly effective while nonintegrative AAV provides a safer means for potential therapeutic applications [33-35]. To take advantage of both systems, we designed and constructed two sets of Cre-recombinase expression vectors. To enable live cell monitoring and facilitate engineered cell enrichment, we further designed both vector sets with either green fluorescent protein (GFP) and/or Puromycin-resistant gene (Puro).

Here, we report the development and potential use of two novel viral-based Cre-delivery systems in cultured mammalian cells. We validated the ability of these two Cre-delivery systems in the removal of Floxed sequences using our previously established TALEN-edited human HEK293 cells [28]. Our study demonstrates that the floxed reporter genes introduced by targeted nucleases and the homologous donor can be successfully removed by viral delivery of Cre-recombinase in HEK293 cells. This new resource will provide researchers a robust and easy-to-use method to conduct more complicated and advanced genome engineering.

\section{Results and discussions}

\section{Design and construction of Cre-delivery vectors}

In order to establish a robust and easy-to-use Credelivery system for gene editing, we used three state-ofthe-art technologies in this study: i) the precise cutting ability of targeted nucleases (TALEN) for doublestranded DNA [28]; ii) the site-specific recombinase of Cre-LoxP, and iii) two powerful viral gene delivery systems, lentivirus and AAV delivery.

First, we designed and constructed five lentiviral vectors for expressing Cre recombinase (Cre) with single or dual promoters (Fig. 1 a-e). One vector contains a CMV promoter driving Cre (Cre sequences in Additional file 1) expression and a WPRE at the 3'end of the construct (Fig. 1a). Three vectors are dual promoter constructs with CMV driving Cre expression and EF1 $\alpha$ driving GFP and/or puro with WPRE at the $3^{\prime}$ end of the marker gene (Fig. 1b-d). The fifth construct is an inducible vector where Cre was cloned into the All-in-one Cumate switch inducible lentivector (Fig. 1e). In this Cumate inducible vector, Cre is driven by the Cumate Operator $(\mathrm{CuO})$ which is turned on when Cumate is added (System Bioscience Inc. (SBI), Palo Alto, CA), while EF1 drives expression of the CymR repressor and Puromycin separated by a ribosomal skip site T2A (Fig. 1e). For lentiviral vectors, the WPRE mRNA stabilization sequence was placed $5^{\prime}$ to the poly-A signal.

Using a similar strategy, the AAV vectors for expressing Cre are configured from $5^{\prime}$ to $3^{\prime}$ as follows: $5^{\prime}$ inverted terminal repeat (ITR), a constitutive cytomegalovirus promoter (CMV), Cre, a poly A signal (poly-A), and 3'ITR (Fig. 1f-i). The marker genes GFP and/or Puro for live cell monitoring and/or drug selection respectively are included under the constitutive EF1 $\alpha$ promoter (Fig. 1f-i). T2A is placed between GFP and puro in constructs containing both markers.

The authenticity and accuracy of all these expression vectors were verified by full sequencing prior to functional testing. 


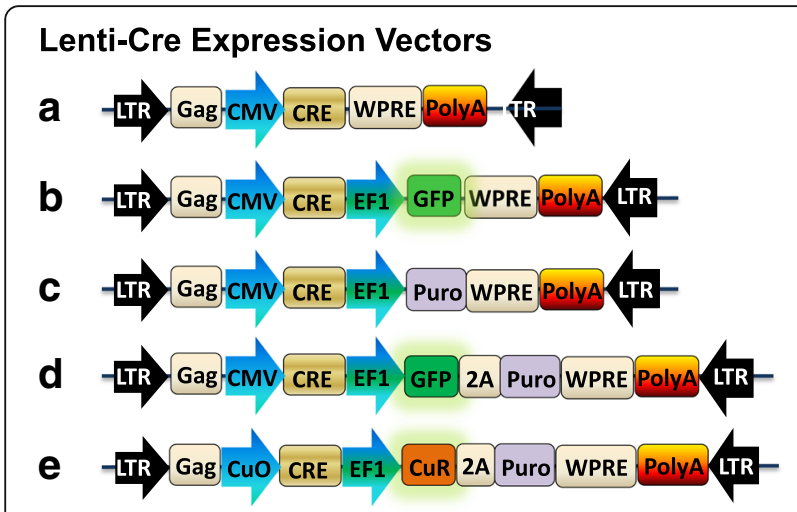

AAV-Cre Expression Vectors
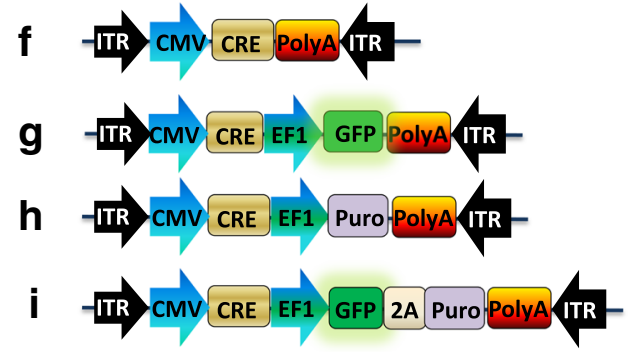

Fig. 1 Schematic illustration of design and construction of recombinant $A A V$ and lentiviral vectors for Cre expression. Features of lentiviral vectors, depicting the humanized Cre gene under the control of the CMV promoter without a selection marker (a) or in a combination with optional selection markers, including GFP (b), Puro (c), GFP plus Puro (d). The Cumate-inducible expression of CRE using an all-in-one lentiviral vector (e). Similarly, features of the AAV vectors, depicting the humanized Cre gene under the control of the CMV promoter alone (f) for with optional selection markers GFP $(\mathbf{g})$, Puro (h), or GFP plus Puro (i)

\section{System setup for functional testing of Cre-delivery} vectors

Targeted nucleases have been successfully used to knock in or knock out genes for cancer research, developmental studies and gene therapy, and can produce bi-allelic changes via their robust nuclease activities $[2,5,8]$. We previously accomplished bi-allelic gene ablation of MIR-21 gene using a TALEN pair and a homologous recombination donor in HEK293 cells (Fig. 2a) [28]. Using this established TALENengineered HEK293 cells (HEK293-TE), in this study we developed a strategy for monitoring Cre activities for our newly designed and constructed Cre expression vectors. This reporting strategy is critical and highly relevant to our study, as it clearly demonstrates the power of combining targeted nucleases technology with Cre-LoxP and viral delivery system to fulfill advanced genome editing.

To test the feasibility of this approach, we transfected red fluorescent HEK293-TE cells with a lentivector expressing both Cre and GFP (Fig. 2b, upper right). Genome typing and sequence analysis show an indel on one allele and a knockin and knockout change on the other allele in one HEK293-E clone (Fig. 2a upper and middle panels; Fig. c). By visual observation, a fraction of cells showed green fluorescence on Day 1 following the transfection, indicating a fast onset of transgene (Cre) expression using a transient transfection protocol. On Day 2, the green fluorescence became stronger while the red fluorescence became weaker, suggesting the removal of floxed RFP-Puro from the engineered cells. On Day 3, cells expressing Cre-GFP become evident and distinctive when comparing fluorescence signals of RFP, as recorded in Fig. 2b (upper left). The red and green fluorescence do not overlap in the same field, indicating that these cells still express either the floxed-RFP-Puro or Cre-GFP (Fig. 2b, lower panels). We contribute the mutual exclusive phenomena to the successful delivery and expression of robust Cre gene and the subsequent removal of floxed-RFP-Puro sequences. This transfection data shows the effectiveness of Cre-LoxP technology.

Next, we verified the removal of floxed RFP-Puro in HEK293-TE reporter cells by genome typing analysis. In both TALEN- and Cre-engineered cells, the indel loci ( $\sim 30 \mathrm{bp})$ should be present at all times, representing the PCR product of NHEJ-modified allele. Under our PCR condition, the knockin allele is intended not to be amplified by limiting the extension time of PCR. Thus, while TALEN-engineered cells will show one PCR product, the Cre-engineered cells is expected to show two PCR products, including the indel $(\sim 430 \mathrm{bp})$ and successful removal of knockin floxed RFP-Puro cassette $(\sim 350 \mathrm{bp})$. As predicted, the knockout loci (KO, $\sim 350 \mathrm{bp}$ ) appears only after the introduction of Cre into the cells (CRE+ lanes), and represents the removed region of the floxed-RFP-Puro cassette (Fig. 2c). These results confirm that Cre is editing out floxed sequences in engineered cells. Additionally, this fluorescent toggling provides an easy way to real time monitor the activities of Cre in live mammalian cells. Therefore, we used the fluorescence features of these reporter cells to conduct the rest of our studies with microscope.

\section{Lenti-Cre efficiently removes floxed RFP-puro in HEK293-}

\section{TE reporter cells}

Previously we and others demonstrated that the vesicular stomach virus envelope glycoprotein (VSVG)pseudotyped lentiviruses have a super high infection rate of $\sim 100 \%[36,37]$. In this study we chose VSVGpseudotyping protocol for lenti-Cre virus production, and examined whether VSVG-pseudotyped lenti-CreGFP could deliver, express and subsequently edit the knockin floxed-RFP-Puro in reporter cells. We transduced HEK293-TE reporter cells with a low MOI of 


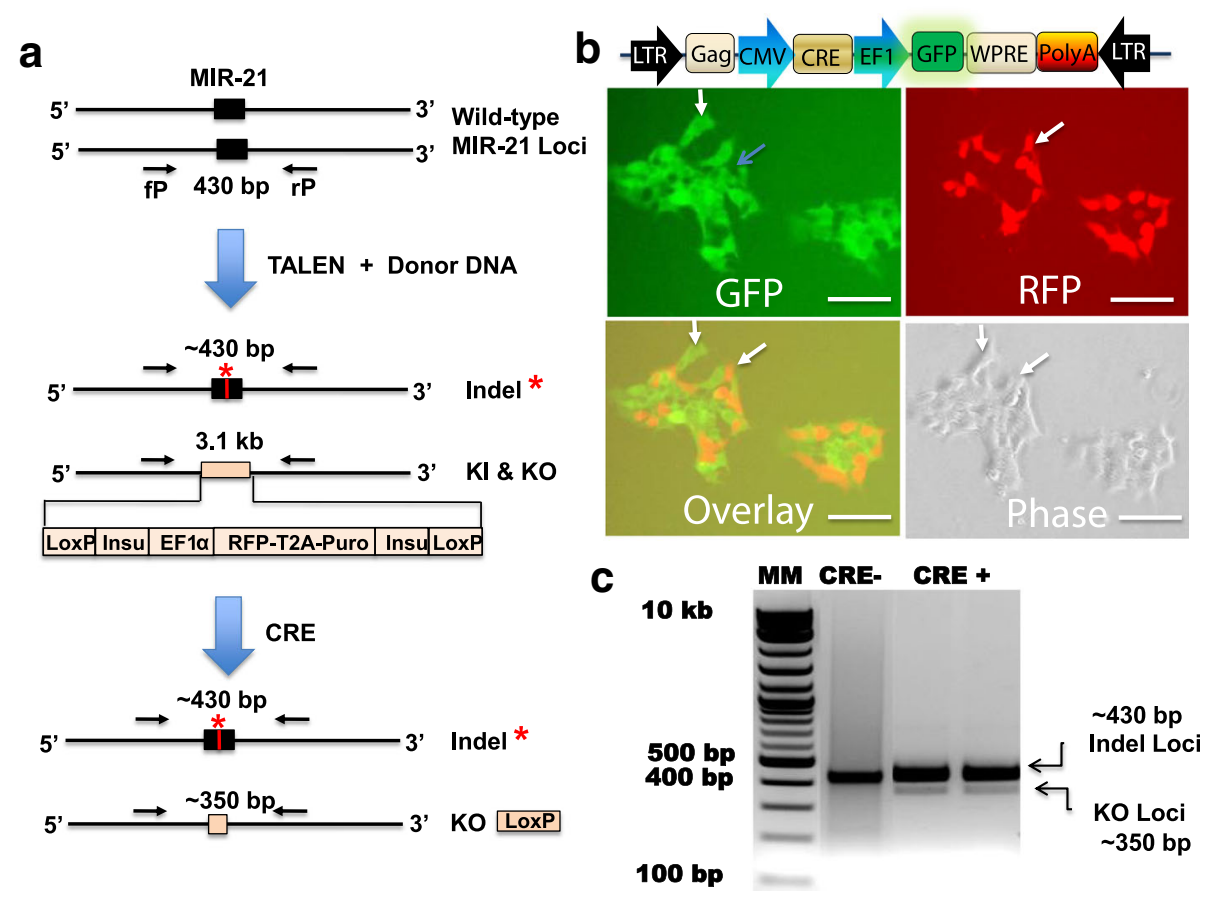

Fig. 2 Strategy for establishing TALEN-engineered HEK293 reporter for testing Cre function. a The schematic flowchart shows the unmodified HEK293 cells harboring the wild-type MIR-21 gene at both loci (upper panel). Introduction of TALEN and HR donor DNA results in bi-allelic ablation of MIR-21 [28]. Genome sequence confirmed an indel of $5 \mathrm{bp}$ at one allele and a knockin (KI) and knockout (KO) event at the other allele via homologous recombination (middle panel). These TALEN-engineered HEK293 (HEK293-TE) cells may serve as a reporter cell line for functional test of the CRE activities (lower panel). b The lenti-Cre-GFP vector is introduced into the HEK293-TE cells by transfection. Green fluorescence shows the expression of Cre-GFP (upper left) and red fluorescence shows the expression of floxed-RFP-Puro (upper right). The overlay shows that the CreGFP expressing cells do not overlap with the Floxed-RFP-Puro cells(lower left). c Genotyping of the HEK293-TE cells after Cre-GFP introduction. The upper $\sim 430 \mathrm{bp}$ is the indel allele and lower $\sim 350 \mathrm{bp}$ is corresponding to the smaller PCR product resulting from the removal of the floxed reporter cassette. The Foxed reporter cassette $(3.1 \mathrm{~kb}$ ) allele could not be amplified under our experimental conditions. White arrows show GFP and RFP positivity are mutually exclusive. Scale bar $50 \mu \mathrm{m}$

0.5 to test the efficiency and robustness of lentisystem. As predicted, we initially observed a weak transgene expression (GFP) at Day 2 after viral transduction. The GFP signal became stronger and evident on Day 3, indicating a relative slower process of transgene expression compared to the transfection protocol described above. In the meantime, the RFP signal became weaker in the GFP positive cells. On days 5-7, fluorescent signals became strong and distinctive for either GFP or RFP (Fig. 3a-c). The mutual exclusiveness of GFP and RFP fluorescence showing in these lentiviral infection data demonstrates the robust and efficient gene editing of this lenti-Cre delivery system. Under the same experimental condition, another lenti-Cre-GFP-Puro vector demonstrates the equivalent activity in the removal of floxed RFP-Puro in a similar fashion (Fig. 3d-f). These results lead to two important conclusions: first, lenti-delivery to recipient cells is robust; second, the onset of Creediting may be delayed as compared to that of transfection protocol. The delay is most likely the time it takes for the lenti-Cre to integrate into the host genome. In transfection, however, the expression of Cre starts immediately because they are independent of integration. In summary, transfection protocol has a rapid onset of transgene expression with moderate transfection rate, which may be suitable for easy-to-transfect cell types, while transduction protocol has a delayed onset of transgene expression with a high transduction rate, which may be applicable to more difficult-to-transfect cell types. In addition, to take advantage of optional markers, both our lentiCre protocols are easily amenable to fluorescence activated cell sorter (FACS) or simple drug selection protocol. Together, our lenti-Cre system provides a robust and flexible solution to advanced genome editing in mammalian cells.

AAV-Cre removes floxed RFP using both transfection and transducing protocols in HEK293-TE reporter cells

In contrast to integrated lentivirus, AAV allows delivery of transgenes into cells without integrating into 

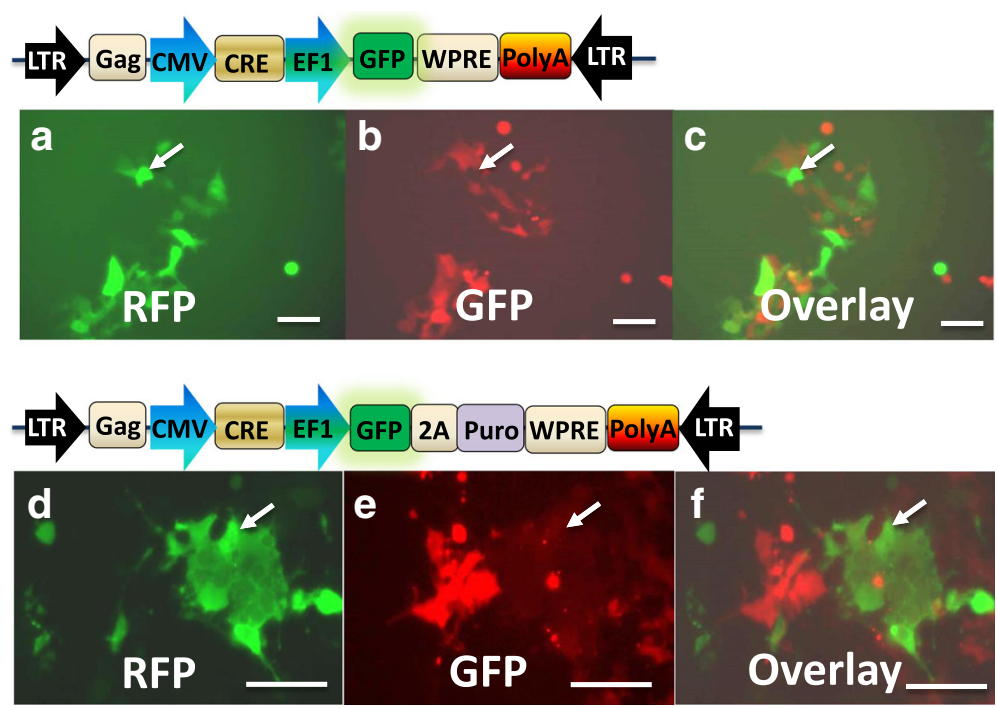

Fig. 3 Efficient removal of floxed-reporter genes with lentiviral transduction protocol. Functional validation of Cre-expression vectors using viral transduction. HEK293-TE cells at 20 40\% confluency were transduced (0.5 MOl)with either lenti-Cre-GFP (a-c) or lenti-Cre-GFP-Puro (d-e) for 5 days and images were taken at 20x magnification. Green fluorescence indicates the expression of Cre-GFP or Cre-GFP-Puro (a, d). Red fluorescence indicates the expression of floxed-RFP-puro (b, e). Overlay of either A and B, or D and E, respectively, shows that the Cre-GFP expressing cells do not overlap with the floxed-RFP (c) or floxed-RFP-puro (f) cells. White arrows show GFP and RFP positivity are mutually exclusive. Scale bar $50 \mu m$

the host cell genome. We next studied genomeediting activities of AAV-Cre in HEK293-TE reporter cells. First, we tested whether AAV vectors can be used by simple transfection protocol. As shown in Fig. 4, transfection with AAV-Cre-GFP-Puro resulted in the appearance of GFP in a fraction of cells (Fig. 4a), parallel with the disappearance of RFP in the same cell population (Fig. 4b-d), suggesting the successful removal of floxed RFP-Puro from the engineered cells by introduction of Cre gene into the cells.

The success in our AAV-Cre transfection protocol encouraged us to further examine the performance of these vectors on HEK293-TE cells by transduction protocol. Despite the use of AAV technology in vivo, the infection rate of AAV is not as efficient as lenti virus and is relatively low in in vitro cell culture conditions. However, since AAV has low toxicity, we transduced reporter cells with high dose of viruses (2 MOI; using either Cre-GFP editing or GFP nonediting control viruses). Consistent with a weaker infectivity under cell culture conditions, the expression of transgene GFP appears on Day 3 and became stronger on Days 4 5 (Fig. 4, e-j). The disappearance of RFP however, occurred on Days 6 7 after transduction (data not shown). On Day 10, almost all cells become GFP-positive and RFP-negative in the Cre group (Fig. 4e-g), suggesting the successful removal of floxed RFP-Puro from HEK293-TE cells transduced with Cre-GFP editing virus. In contrast, cells become GFP-positive (Fig. 4h) but remain RFP-positive (Fig. j) in GFP non-editing control group. Together, we validated the AAV-Cre delivery system using both easy-to-perform transfection and viral transduction protocols. The non-integrative nature of AAV makes it particularly useful for clinical applications.

Lenti- and AAV-based systems provide a comprehensive solution to advanced genome editing in various mammalian cells

In this study, we explored the combination of three advanced technologies: targeted nucleases, Cre-LoxP and lentiviral/AVV delivery systems, and successfully established a robust and easy-to-use Cre-delivery system for gene editing in mammalian cells. Targeted nucleases have been the core technologies to knockin or knockout genes for developmental studies, cancer research and gene therapy $[10,13]$. Genome modifications producing bi-allelic changes can be made through targeted genome cleavage by engineered, sequence-specific nucleases such as TALENs [2]. CreLoxP is another genome editing mechanism with unique advantages. First, it is a simple two-factor system requiring only the Cre recombinase enzyme and two LoxP sites. Second, it can be used in any cellular environment including mammalian cells, yeast cells, and has applications both in vitro and in vivo. Third, it also can be made conditionally by incorporating to 

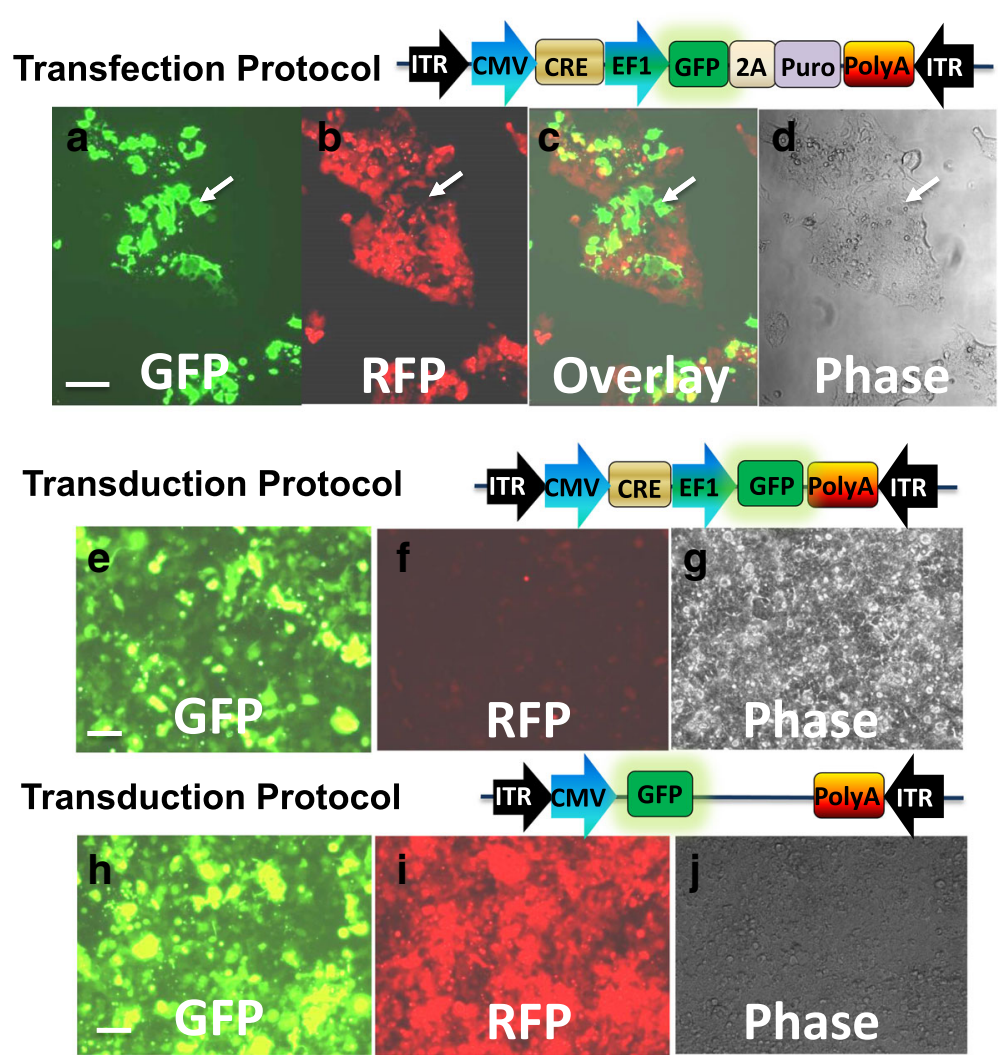

Fig. 4 Functional Validation of AAV-based Cre in engineered HEK293 cells. HEK293-TE cells were transfected with the constructs expressing Cre and GFP (a-d). Images were taken at 20x magnifications, 3 days after transduction. Green fluorescence depicts cells expressing CreGFP-puro (a). Red fluorescence depicts cells expressing floxed-RFP-puro (b). Overlay of the left and middle images, respectively shows that cells either express RFP or GFP (c), and the phase contract of overlay (d). Alternatively, cells at 20 30\% confluency were transduced with the AAV (2.0 MOI) expressing Cre-GFP (e to $\mathbf{g}$ ) or the control GFP viruses (h to $\mathbf{j}$ ). Images were taken at 20x magnification, 10 days after transduction Green fluorescence indicates the expression of Cre-GFP (e) or control GFP (h). Diminished red fluorescence indicates the expression of floxed-RFP-puro (f). In contrast, red fluorescence signal remains strong in GFP non-editing control (i). Phase contrast shows the TALEN-Cre sequential edited HEK293 cells (g) vs. control cells (j). White arrows show that GFP and RFP positivity are mutually exclusive. Scale bar $50 \mu \mathrm{m}$

an inducible vector such as Cumate-operator system. Finally, the Cre-LoxP system can work in quick, effective and precise manners by transfection protocols in easy-to-transfect cell line such as HEK293 cells.

Previously we had developed a system for delivering various genes into cells using either lentiviral or AAV particles $[36,38]$. We initially selected the lentiviral system because of its favorable features: (1) Lentiviral transduction is an efficient way to create a stable cell line expressing Cre in almost any mammalian cell type including stem cells and non-dividing cells. Results from these studies indicate that the onset of expression and removal of floxed RFP in lentiviral transduced cells is faster than in AAV (5 vs. 7-10 days). (2) Lentiviral system can be customized with a Cumate-inducible promoter driving expression of $\mathrm{Cre}$, enabling scientists to follow biological changes induced by Cre in real time, thus expanding its application (Additional file 2: Figure S1). (3)
There is a newly available integrase-defective lentiviral packaging system, which enables transduction of larger lentiviral constructs without undesired integration of the lenti construct into the genome [36]. In contrast, AAV vectors have a smaller gene packaging size of $\sim 3.7 \mathrm{~kb}$, which make them unsuitable for building all-in-one inducible Cre ( 7.0 kb in size). However, AAV system has its own advantages: (1) they can easily deliver Cre into their target cells without integration into the host cell genome, thus enabling experiments targeted for potential clinical applications such as gene therapy or therapeutic purposes. To make use of the packaging capacity of lentivector, we have built a full suite of expression vectors with/without selection makers GFP and/or Puro (Additional file 3: Figure S2A-C), thus providing options that can meet different research needs. Furthermore, the broad tropism (Additional file 3: Figure S2D-G) coupled with titratable feature of AAV-based vectors (Additional file 3: Figure 
S2h-k) make them desirable for delicate applications with less genome toxicity [39].

\section{Conclusions}

We developed and functionally validated two effective viral Cre-delivery systems with two different flavors (Lenti and AAV) for advanced genome-editing in mammalian cells. These novel methods combine sequence-specific editing ability of Cre with targeted nucleases, which enables complicated genome manipulation in a vast variety of cell types. The built-in reporters and inducible elements designed in the lenti and AAV vectors enable live cell monitoring, drug selection and temporal knockout. Although both CRISPR-Cas9 and TALEN technologies are getting more popular, their ability in editing floxed sequences is limited and thus requires Cre to carry out specific action under these situations. The precise sequence editing ability of Cre coupled with efficient delivery to various mammalian cell types make these systems very attractive, rendering broad applications and generating sophisticated but biologically relevant results.

\section{Materials and methods}

Design and construction of lentiviral and AAV vectors for Cre-recombinase

Five lentiviral vectors expressing Cre recombinase (Cre) were designed and constructed with single or dual promoters (Fig. 1A-E). Using similar strategy, five AAV vectors for expressing Cre recombinase were designed and constructed (Fig. $1 \mathrm{f}-\mathrm{i}$ ). The construction of all vectors was performed by PCR amplification of fragments and were joined together using a seamless cloning kit (SBI, Cat\# MC100A-1) as reported previously [40]. All final constructs were verified by full sequencing.

\section{Cell culture, transfection, and Cumate induction}

Human embryonic kidney cells (HEK293) were cultured and maintained in high glucose Dulbecco's Minimal Essential Medium (DMEM) supplemented with 10\% FBS, $2 \mathrm{mM}$ GlutaMax, $100 \mathrm{U} / \mathrm{ml}$ penicillin and $100 \mathrm{U} / \mathrm{ml}$ streptomycin. The engineered cells expressing RFP and puromycin resistance were maintained in the presence of $5 \mu \mathrm{g} / \mathrm{mL}$ puromycin but switched to normal complete medium at least 2 passages before experimentation. Two different HEK293 cells were used in this study: TALEN-engineered HEK293 expressing RFP (HEK-293TE) [28] and 293TN cells (SBI, Cat\# LV900A1) for viral packaging and AAV titration.

All transfections were performed in 6-well plates. Cells were plated at a density of $2 \times 10^{5}$ cells per well on the day before transfection. Cells were at $30 \sim 50 \%$ confluency the next day and transfected with plasmid
(2 $\mu \mathrm{g}$ ), using Purefection transfection reagent (SBI, Cat\#LV850A-1) according to manufacturer's instructions. For virus transduction, cells were plated at a lower density of $0.5 \times 10^{5}$ cells per well on the day before transduction. Cells expressing inducible Cre constructs were treated with $5 \times$ Cumate for induction experiments (SBI, Cat\#QM100A-1).

\section{Lentiviral packaging and titration}

Lentiviral vector constructs were packaged into lentivirus using pPACKH1 (SBI, Cat\# LV500A-1) as previously reported [36, 41]. 293TN cells were transfected with lenti-vector and pPACK-H1, lentivirus packaging mix. 48 and $72 \mathrm{~h}$ post transfection, cell culture media containing Lentivirus was collected at and concentrated using PEG-it (SBI, Cat\# LV810A$1)$. The viral pellet was suspended in sterile PBS and tittered using the Global Ultra-Rapid Tattering kit (SBI, Cat\# LV961A-1). These concentrated lentiviruses were used to infect the $293 \mathrm{TN}$ cells.

\section{AAV production and titration}

293TN cells were plated in a $10 \mathrm{~cm}$ dishes to reach a confluency of $70 \sim 80 \%$ in DMEM growth media with $10 \%$ FBS and 5\% Glutamax overnight. Cells were transfected the next day with $12 \mu \mathrm{g}$ each of the following: AAV vector, pAAV-RC (Cell Biolabs, Inc., San Diego, Cat\# VPK-422) and AAV helper plasmids (Cell Biolabs, Inc., Part No.340202) at a 1:1:1 ratio, for a total of $36 \mu \mathrm{g}$ of plasmid DNA., The media was changed to complete DMEM with Penn/Strep 18 to $24 \mathrm{~h}$ after transfection. $72 \mathrm{~h}$ post transfection, the media was collected and briefly spun down at $3000 \mathrm{rpm}$ for 15 min to get rid of cell debris. AAVanced ${ }^{\text {mix }}$ Concentration Reagent (SBI, Cat\# AAV100A-1) was added according to the manufacturer's instructions. The viral pellet was re-suspended in a small volume of sterile PBS. This concentrated AAV was used to infect the HEK293-TE cells. AAV-DJ helper free packaging system was used unless otherwise indicated.

Both green cell fluorescent assays and PCR were used to determine the multiple of infection (MOI) as reported [38]. For the green cell fluorescent assays, 293TN cells grown on 12-well plates were infected with serial dilutions of CMV-GFP-2A-Luciferase positive control virus. $72 \mathrm{~h}$ later, cells infected with GFP-positive virus were visually scored using a fluorescence microscope, and the viral MOI was determined by GFP positive cells. The MOI of AAVreporter was estimated by the relative copy number of recombinant virus versus the positive control viruses as reported [42, 43]. 


\section{Genotyping to confirm the removal of floxed RFP-puro re- porter genes from genome}

To confirm the removal of floxed RFP-puro (3.1 kb), the MIR-21 gene loci were PCR-amplified from genomic DNA of Cre-transduced cells and the control cells using the EZ Genotyping kit (Cat\# GE200A-1,SBI) according to the manufacturer's instructions. To determine genome types, a pair of primers (forward primer, fP: $5^{\prime}$ TGGGGTTCGATCTTAACAGG-3' and reverse primer, rP: 5' -TTTCAAAACCCACAATGCAG-3') was used to perform PCR on the MIR-21 loci. PCR products with sizes of $430 \mathrm{bp}$ for the indel allele or $350 \mathrm{bp}$ for the floxed-RFP-puro cassette-removed allele were selectively amplified under the condition: $94{ }^{\circ} \mathrm{C}, 30 \mathrm{~s} ; 60{ }^{\circ} \mathrm{C}, 30 \mathrm{~s}$; and $72{ }^{\circ} \mathrm{C}, 10 \mathrm{~s}$, for a total of 30 cycles. The products were then subject to $2 \%$ agarose gel electrophoresis in $1 \times$ TAE buffer.

\section{Microscopy and live cell monitoring}

All microscopy was performed on live cells using a LEICA DMI3000B fluorescent microscope. Data collection and processing were performed with LAS 3.8 software. The same field was subject to imaging under different conditions such as phase contrast, GFP and/or RFP. Imaging was further processed and overlaid using Adobe Photoshop CS or MS PowerPoint program to illustrate the relationships of GFP and/or RFP positivity.

\section{Additional files}

Additional file 1: Coding and amino-acid sequences of Cre recombinase. (PDF $40 \mathrm{~kb}$ )

Additional file 2: Figure S1. The conditional removal of floxed RFP from HEK293 genome by Cumate-induction. (PDF 412 kb)

Additional file 3: Figure S2. Features of AAV-based Cre-delivery vectors. (PDF $367 \mathrm{~kb}$ )

\section{Abbreviations \\ CMV: cytomegalovirus promoter; EF1a: Elongation Factor 1-alpha promoter; FBS: fetal bovine serum; GFP: green fluorescent protein; HEK293: human embryonic kidney cell line; HEK293-TE: TALEN-engineered HEK293 cell; ITR: inverted terminal repeats; LTR: long terminal repeats; mCMV: minimal CMV promoter; Puro: puromycin-resistant gene; RFP: red fluorescent protein; T2A: self-cleaving 2A peptide sequence; TALEN: Transcription activator-like ef- fector nuclease; TF: transcription factor}

\section{Acknowledgements}

We would like to thank members of the System Bioscience Inc. for their technical assistance. We also thank Dr. Claudia Uhde-Stone for helpful discussion.

\section{Funding}

This work was supported by R\&D fund from System Biosciences, a start-up fund from the Department of Bioengineering.

\section{Availability of data and materials}

The sequence data can be retrieved from the additional file and all vector constructs can be obtained from SBI.

\section{Authors' contributions}

$\mathrm{BL}$ and NS designed and executed experimental approaches, performed experiments, analyzed data, coordinated the team project, and wrote the manuscript. RS and AM planned experimental approaches, analyzed data and wrote the manuscript. CM and AA performed the experiments and participate in writing the manuscript. $\mathrm{JH}$ helped to interpret data and revised the manuscript

Ethics approval and consent to participate

Not applicable.

\section{Consent for publication}

Not applicable.

\section{Competing interests}

$\mathrm{RS}, \mathrm{AM}, \mathrm{CM}, \mathrm{AA}, \mathrm{JH}$ declare financial competing interest as System Biosciences (SBI) employee. NS and BL were previous employees of SBI.

\section{Publisher's Note}

Springer Nature remains neutral with regard to jurisdictional claims in published maps and institutional affiliations.

\section{Author details}

'System Biosciences, 2436 Embarcadero Way, Palo Alto, CA 94303, USA. ${ }^{2}$ Gilead Sciences Inc., 333 Lakeside Drive, Foster City, CA 94404, USA.

${ }^{3}$ Department of Bioengineering, Santa Clara University, 500 El Camino Real, Santa Clara, CA 95053, USA.

Received: 22 September 2017 Accepted: 14 November 2017

Published online: 24 November 2017

\section{References}

1. Stoddard BL. Homing endonucleases: from microbial genetic invaders to reagents for targeted DNA modification. Structure. 2011;19(1):7-15.

2. Gaj T, Gersbach CA, Barbas CF 3rd, ZFN TALEN. CRISPR/Cas-based methods for genome engineering. Trends Biotechnol. 2013;31(7):397-405.

3. Hsu PD, Lander ES, Zhang F. Development and applications of CRISPR-Cas9 for genome engineering. Cell. 2014;157(6):1262-78.

4. Miller JC, Tan S, Qiao G, Barlow KA, Wang J, Xia DF, Meng X, Paschon DE, Leung E, Hinkley SJ, et al. A TALE nuclease architecture for efficient genome editing. Nat Biotechnol. 2010;29(2):143-8.

5. Segal DJ, Meckler JF. Genome engineering at the dawn of the golden age. Annu Rev Genomics Hum Genet. 2013;14:135-58.

6. Cong L, Ran FA, Cox D, Lin S, Barretto R, Habib N, Hsu PD, Wu X, Jiang W, Marraffini LA, et al. Multiplex genome engineering using CRISPR/Cas systems. Science. 2013;339(6121):819-23.

7. Koike-Yusa H, Li Y, Tan EP, Velasco-Herrera Mdel C, Yusa K. Genome-wide recessive genetic screening in mammalian cells with a lentiviral CRISPRguide RNA library. Nat Biotechnol. 2014;32(3):267-73.

8. Gersbach CA. Genome engineering: the next genomic revolution. Nat Methods. 2014;11(10):1009-11.

9. Lillico SG, Proudfoot C, Carlson DF, Stverakova D, Neil C, Blain C, King TJ, Ritchie WA, Tan W, Mileham AJ, et al. Live pigs produced from genome edited zygotes. Sci Rep. 2013;3:2847.

10. Osborn MJ, Starker CG, McElroy AN, Webber BR, Riddle MJ, Xia L, DeFeo AP Gabriel R, Schmidt M, von Kalle C, et al. TALEN-based gene correction for epidermolysis bullosa. Mol Ther. 2013;21(6):1151-9.

11. Lieber MR. The mechanism of double-strand DNA break repair by the nonhomologous DNA end-joining pathway. Annu Rev Biochem. 2010; 79:181-211

12. Mahfouz MM, Li L, Shamimuzzaman M, Wibowo A, Fang X, Zhu JK. De novo-engineered transcription activator-like effector (TALE) hybrid nuclease with novel DNA binding specificity creates double-strand breaks. Proc Natl Acad Sci U S A. 2011;108(6):2623-8.

13. Schwank G, Koo BK, Sasselli V, Dekkers JF, Heo I, Demircan T, Sasaki N, Boymans S, Cuppen E, van der Ent CK, et al. Functional repair of CFTR by CRISPR/Cas9 in intestinal stem cell organoids of cystic fibrosis patients. Cell Stem Cell. 2013;13(6):653-8.

14. Wu Y, Liang D, Wang Y, Bai M, Tang W, Bao S, Yan Z, Li D, Li J. Correction of a genetic disease in mouse via use of CRISPR-Cas9. Cell Stem Cell. 2013; 13(6):659-62. 
15. Long C, McAnally JR, Shelton JM, Mireault AA, Bassel-Duby R, Olson EN. Prevention of muscular dystrophy in mice by CRISPR/Cas9-mediated editing of germline DNA. Science. 2014;345(6201):1184-8.

16. Soldner F, Laganiere J, Cheng AW, Hockemeyer D, Gao Q, Alagappan R, Khurana V, Golbe LI, Myers RH, Lindquist S, et al. Generation of isogenic pluripotent stem cells differing exclusively at two early onset Parkinson point mutations. Cell. 2011;146(2):318-31.

17. Hockemeyer D, Soldner F, Beard C, Gao Q, Mitalipova M, DeKelver RC, Katibah GE, Amora R, Boydston EA, Zeitler B, et al. Efficient targeting of expressed and silent genes in human ESCS and iPSCs using zinc-finger nucleases. Nat Biotechnol. 2009;27(9):851-7.

18. Hockemeyer D, Wang H, Kiani S, Lai CS, Gao Q, Cassady JP, Cost GJ, Zhang $L$, Santiago $Y$, Miller JC, et al. Genetic engineering of human pluripotent cells using TALE nucleases. Nat Biotechnol. 29(8):731-4.

19. Sun $\mathrm{N}$, Zhao H. Seamless correction of the sickle cell disease mutation of the HBB gene in human induced pluripotent stem cells using TALENs. Biotechnol Bioeng. 2013;111(5):1048-53.

20. Sauer B, Henderson N, Site-specific DNA. Recombination in mammalian cells by the Cre recombinase of bacteriophage P1. Proc Natl Acad Sci U S A. 1988;85(14):5166-70.

21. Orban PC, Chui D, Marth JD. Tissue- and site-specific DNA recombination in transgenic mice. Proc Natl Acad Sci U S A. 1992;89(15):6861-5.

22. Gu H, Zou YR, Rajewsky K. Independent control of immunoglobulin switch recombination at individual switch regions evidenced through Cre-loxPmediated gene targeting. Cell. 1993;73(6):1155-64.

23. Gu H, Marth JD, Orban PC, Mossmann H, Rajewsky K. Deletion of a DNA polymerase beta gene segment in T cells using cell type-specific gene targeting. Science. 1994;265(5168):103-6.

24. Turan S, Galla M, Ernst E, Qiao J, Voelkel C, Schiedlmeier B, Zehe C, Bode J. Recombinase-mediated cassette exchange (RMCE): traditional concepts and current challenges. J Mol Biol. 2011;407(2):193-221.

25. Sternberg N, Hoess R. The molecular genetics of bacteriophage P1. Annu Rev Genet. 1983;17:123-54

26. Nagy A. Cre recombinase: the universal reagent for genome tailoring Genesis. 2000;26(2):99-109.

27. Zheng B, Sage M, Sheppeard EA, Jurecic V, Bradley A. Engineering mouse chromosomes with Cre-loxP: range, efficiency, and somatic applications. Mol Cell Biol. 2000;20(2):648-55

28. Uhde-Stone C, Sarkar N, Antes T, Otoc N, Kim Y, Jiang YJ, Lu B, TALEN-based A. Strategy for efficient bi-allelic miRNA ablation in human cells. RNA. 2014; 20(6):948-55.

29. Ho TT, Zhou N, Huang J, Koirala P, Xu M, Fung R, Wu F, Mo YY. Targeting non-coding RNAs with the CRISPR/Cas9 system in human cell lines. Nucleic Acids Res. 2015;43(3):e17.

30. Matrai J, Chuah MK, VandenDriessche T. Recent advances in lentiviral vector development and applications. Mol Ther. 2010;18(3):477-90.

31. Collins M, Thrasher A. Gene therapy: progress and predictions. Proc Biol Sci. 2015;282(1821):20143003

32. Yang X, Boehm JS, Salehi-Ashtiani K, Hao T, Shen Y, Lubonja R, Thomas SR, Alkan O, Bhimdi T, Green TM, et al. A public genome-scale lentiviral expression library of human ORFs. Nat Methods. 2011;8(8):659-61.

33. McCarty DM, Monahan PE, Samulski RJ. Self-complementary recombinant adeno-associated virus (SCAAV) vectors promote efficient transduction independently of DNA synthesis. Gene Ther. 2001;8(16):1248-54.

34. Santiago-Ortiz J, Ojala DS, Westesson O, Weinstein JR, Wong SY, Steinsapir A Kumar S, Holmes I, Schaffer DV. AAV ancestral reconstruction library enables selection of broadly infectious viral variants. Gene Ther. 2015;22(12):934-46.

35. Ojala DS, Amara DP, Schaffer DV. Adeno-associated virus vectors and neurological gene therapy. Neuroscientist. 2014;21(1):84-98.

36. Sengupta R, Mukherjee C, Sarkar N, Sun Z, Lesnik J, Huang J, Lu B. An optimized protocol for packaging Pseudotyped integrase defective lentivirus. Biol Proced Online. 2016;18:14.

37. Stegmeier F, Hu G, Rickles RJ, Hannon GJ, Elledge SJ. A lentiviral microRNAbased system for single-copy polymerase II-regulated RNA interference in mammalian cells. Proc Natl Acad Sci U S A. 2005;102(37):13212-7.

38. Zhang Z, Stickney Z, Duong N, Curley K, Lu B. AAV-based dual-reporter circuit for monitoring cell signaling in living human cells. J Biol Eng. 2017;11:18.

39. Choi JH, NK Y, Baek GC, Bakes J, Seo D, Nam HJ, Baek SH, Lim CS, Lee YS, Kaang BK. Optimization of AAV expression cassettes to improve packaging capacity and transgene expression in neurons. Mol Brain. 2014;7:17.
40. Uhde-Stone C, Cheung E, Lu B. TALE activators regulate gene expression in a position- and strand-dependent manner in mammalian cells. Biochem Biophys Res Commun. 2014;443(4):1189-94.

41. Mendenhall A, Lesnik J, Mukherjee C, Antes T, Sengupta R. Packaging HIVor FIV-based lentivector expression constructs and transduction of VSV-G pseudotyped viral particles. J Vis Exp. 2012;62:e3171.

42. Potter M, Lins B, Mietzsch M, Heilbronn R, Van Vliet K, Chipman P, AgbandJemcKenna M, cleaver BD, Clement N, Byrne BJ, et al. a simplified purification protocol for recombinant adeno-associated virus vectors. Mol Ther Methods Clin Dev. 2014;1:14034.

43. Zolotukhin S, Potter M, Zolotukhin I, Sakai Y, Loiler S, Fraites TJ Jr, Chiodo VA, Phillipsberg T, Muzyczka N, Hauswirth WW, et al. Production and purification of serotype 1,2, and 5 recombinant adeno-associated viral vectors. Methods. 2002;28(2):158-67.

\section{Submit your next manuscript to BioMed Central and we will help you at every step:}

- We accept pre-submission inquiries

- Our selector tool helps you to find the most relevant journal

- We provide round the clock customer support

- Convenient online submission

- Thorough peer review

- Inclusion in PubMed and all major indexing services

- Maximum visibility for your research

Submit your manuscript at www.biomedcentral.com/submit
) Biomed Central 